\title{
On Modeling Drilling Load in Lunar Regolith Simulant
}

\author{
Qi-Quan Quan*, Chong-Bin Chen, Zong-Quan Deng, Jun-Yue Tang and De-Wei Tang
}

\begin{abstract}
Drilling and coring, as effective ways to obtain lunar regolith along the longitudinal direction, are widely applied in the lunar sampling field. Conventionally, modeling of drill-soil interaction was divided into soil cutting and screw conveyance processes, ignoring the differences in soil mechanical properties between them. To improve the modeling accuracy, a hypothesis that divides the drill-soil interaction into four parts: cuttings screw conveyance, cuttings extruding, cuttings bulldozing, and in situ simulant cutting, is proposed to establish a novel model based on the passive earth pressure theory. An iterative numerical calculation method is developed to predict the drilling loads. A drilling and coring testbed is developed to conduct experimental tests. Drilling experiments indicate that the drilling loads calculated by the proposed model match well the experimental results. The proposed research provides the instructions to adopt a suitable drilling strategy to match the rotary and penetrating motions, to increase the safety and reliability of drilling control in lunar sampling missions.
\end{abstract}

Keywords: Lunar regolith drilling, Lunar regolith simulant, Drill-simulant interaction, Screw conveyance

\section{Introduction}

Research on lunar regolith can enhance knowledge regarding lunar regolith composition and planetary history $[1,2]$. Drilling systems are widely used for extraterrestrial subsurface regolith sampling, because they are appropriate tools to acquire regolith samples while maintaining their stratification along the longitudinal direction [3-5]. The mission of Chang'e-5 lunar exploration project aims to obtain lunar subsurface regolith through a drilling and coring device, and return it to the Earth. Though the mechanical properties of the lunar regolith obtained by the Soviet Union and the United States have been analyzed and reported in the literature, the regolith materials at a specific site are not known [6,7]. The drill tool should have operative performance to adapt to lunar regolith with different mechanical properties. Therefore, ground experiments must be conducted with the desired lunar regolith simulant to validate whether the drill tool can obtain an appropriate sample [1, 8]. The modeling of the drill-simulant interaction simulates the drilling

*Correspondence: quanqiquan@hit.edu.cn

State Key Laboratory of Robotics and System, Harbin Institute of Technology, Harbin 150001, China mechanism, which is used to predict drilling load, and represents a fundamental reference to eventually design an appropriate drill tool and develop a suitable drilling strategy [9].

The finite element method (FEM) and discrete element method (DEM) have been used to develop the model of drill-soil interaction [9-12]. Because the FEM is a gridbased method, it cannot deal well with large deformation and post-failure problems. It may suffer from mesh distortion, resulting in an inaccurate solution and failure of computational convergence $[1,2]$. Although the DEM does not have that limitation, the high computational cost restricts DEM application to small-scale or shortduration simulations [13].

Analytical models have also been used to predict the drilling load [9, 14-20]. The analytical model of drill-soil interaction was developed using the screw conveyance theory and soil shear rupture principle. The analysis of the soil rupture is based on the passive earth pressure theory, which has successfully been used to predict the bulldozing force [21, 22]. According to the shape and cutting rake angle of the blade, different cutting models have been proposed to describe the soil rupture mechanism [23-26]. These theories can be used as a fundamental
SpringerOpen

(c) The Author(s) 2018. This article is distributed under the terms of the Creative Commons Attribution 4.0 International License (http://creativecommons.org/licenses/by/4.0/), which permits unrestricted use, distribution, and reproduction in any medium, provided you give appropriate credit to the original author(s) and the source, provide a link to the Creative Commons license, and indicate if changes were made. 
reference for modeling drill-tool interaction. The screw conveyance calculation, which is similar to the bulk solid screw conveyor design, can predict particle screw conveyance load and efficiency [27-29]. According to the screw diameters and the rotary speed of an auger, the centripetal force or the weight of particles are the main causes of auger conveyance [30,31]. The rotary speed is always less than $300 \mathrm{r} / \mathrm{min}$, and the diameters of the drill tool are generally limited below $50 \mathrm{~mm}$ in extraterrestrial exploration. For such orders of rotary speed and diameters, the weight of particles could be the main component that provides cuttings conveyance [30]. This study models the screw conveyance process based on a quasi-static analysis. In addition, in a significant number of previous works, the drill-soil interaction was simply divided into screw conveyance and soil cutting processes. The boundary conditions and soil mechanical property differences between these two processes were ignored in previous modeling of the drill-soil interaction. Thus, the calculated results, i.e., the summations of the screw conveyance load and soil cutting load, must either be further verified or only used in special situations.

The authors' previous experiments demonstrated that it is difficult to predict the drilling load accurately when only the screw-conveying load and soil shear rupture are considered during simulant drilling. The simulant rupture is accompanied by simulant chip flow during simulant drilling, which may significantly influence the drilling load. The boundary conditions and simulant mechanical property differences between the simulant cutting and the cuttings conveyance processes should be considered in the drill-simulant interaction model. In this study, according to the structure of the drill tool, the drill-soil interaction is divided into four parts: cuttings conveyance, chip extruding, chip bulldozing, and in situ simulant cutting. To simulate the drilling mechanism, a drill-simulant interaction model, considering the boundary conditions and simulant density difference among these four parts, is developed to predict the drilling load. Drilling experiments are conducted to validate the presented model.

The remainder of this paper is organized as follows. First, the lunar regolith simulant and drill tool used in this paper are introduced. Then, a simulant-cuttings conveyance analysis is presented. Next, a drill-simulant interaction model is developed to predict the drilling load. Finally, drilling experiments are conducted to validate the proposed model and the results are presented.

\section{Lunar Regolith Simulant and Drill Tool}

To sample a lunar regolith along the longitudinal direction, it is necessary to design a special drill tool to adapt to the complex lunar environments. Ground drilling and coring experiments should be conducted with the lunar regolith simulant to validate the performance of the designed drill tool.

\subsection{Lunar Regolith Simulant}

The lunar regolith simulant was created using basaltic pozzolana collected from Nanjing, China. The mineral composition of pozzolana includes plagioclase, olivine, pyroxene, opaque mineral, and volcanic glass, which is similar to that of the lunar regolith from the Apollo 14 landing site [32]. The particle-size range of the simulant is $0.1-1 \mathrm{~mm}$, and the median grain size is $0.41 \mathrm{~mm}$ [33]. According to the data on particle size distribution of lunar regolith, the particle size distribution band of the simulant matches the actual lunar regolith [34, 35].

The relative density of lunar regolith is generally low to medium in the shallow layer, and approaches the maximum value just $10-20 \mathrm{~cm}$ below the lunar surface [6, 36]. Additionally, for a drilling system, the regolith simulant with high relative density is the most challenging case in terms of over-head drilling load [37]. Therefore, the dense regolith simulant may be more suitable to test the drilling device. The dense simulant used in this paper was prepared by vibratory compaction. Physical property tests (following the SL237-1999 standard procedure [38]) reveal that the density, specific gravity, relative density, internal friction angle, and cohesion are $2.14 \mathrm{~g} / \mathrm{cm}^{3}, 2.94 \mathrm{~g} / \mathrm{cm}^{3}, 98 \%, 35.1^{\circ}$, and $2.85 \mathrm{kPa}$, respectively [33].

\subsection{Drill Tool}

To acquire the regolith samples along the depth direction, the drill tool is hollow, as shown in Figure 1, and consists of a drill bit and an auger. The drill bit is used to crack the regolith and maintain the auger position. Four kentanium blades radially mounted on the drill bit are used to cut the regolith. The screw of the auger is used to convey the chips to the lunar surface. A coring device, which has been introduced in the literature, is installed in the hollow auger [33]. Because there is no relative sliding between the regolith and coring device, this coring manner can keep stratification of the regolith and has negligible effect on coring samples [39].

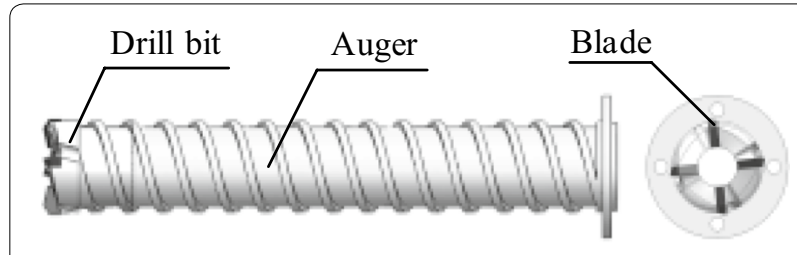

Figure 1 Drill tool 


\section{Simulant Cuttings Conveyance Analysis}

In the authors' previous experiments, the simulant cuttings filled the auger groove when drilling the dense simulant samples with a rotary speed below $200 \mathrm{r} / \mathrm{min}$. However, the density of the chips varies with the drilling parameters (defined as a combination of penetrating speed and rotary speed in this paper) as shown in Figure 2. To explain this conveyance behavior, this section will analyze the screw conveying process, which is the foundation of the drill-soil interaction modeling.

The screw conveyor design assumes that the fictional force created by the centrifugal force is the main component that provides the forward motion of the particles $[22,31]$. The rotary speed is always lower than $300 \mathrm{r} / \mathrm{min}$ (the rotary speed of the CE-5 is limited below $200 \mathrm{r} / \mathrm{min}$ ), and the diameter of the drill tool is generally limited below $50 \mathrm{~mm}$ (the diameter of the CE- 5 is limited below $32 \mathrm{~mm}$ ) in the extraterrestrial exploration. With this order of the rotary speed and diameters, the centrifugal force may not be the main component that provides for the cuttings conveyance [30, 40]. This paper models the screw conveying process based on a quasi-static analysis. In view of the reduced auger groove height and the low motion speed of the chips, the flow of the chips in the auger groove can be assumed to be in the ideal critical state:

(1) The flow of the cuttings is uniform, and the cuttings do not exchange the position in the vertical direction;

(2) The granular vortex motion is ignored.

The in situ simulant is stirred by the drill bit into loose simulant cutting and conveyed to the surface where the drill tool begins to drill into the simulant. The remainder is collected into the sampling device. The penetrating
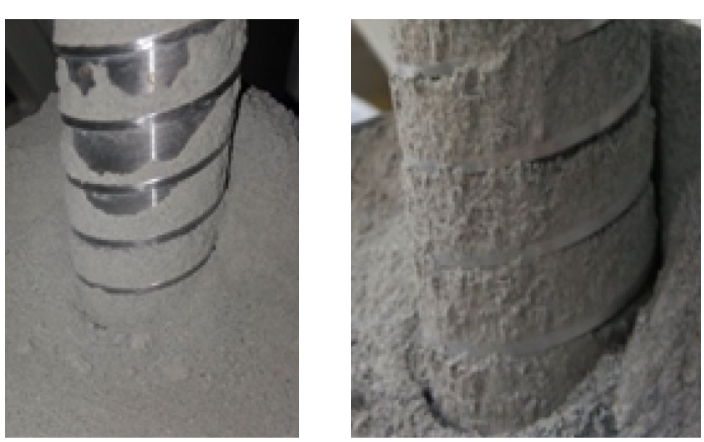

a Rotary speed: 200 r/min b Rotary speed: 85 r/min

Figure 2 Simulant chips in the auger groove under different drilling parameters (Penetrating speed: $85 \mathrm{~mm} / \mathrm{min}$ ) motion is accompanied by the production of cuttings, and the rotary motion conveys the cuttings.

Based on the assumption of the chip flow, the kinematic analysis of the conveying action is depicted in Figure 3 . According to the velocity diagram, the velocity of the simulant chips can be expressed as

$$
\left\{\begin{array}{l}
v_{\mathrm{r}}=\frac{v \sin \beta}{\sin (\alpha+\beta)}, \\
v=\omega r_{1}=2 \pi n r_{1}, \\
v_{\mathrm{s}}=\frac{v \sin \alpha}{\sin (\alpha+\beta)},
\end{array}\right.
$$

where $v$ is the velocity of the auger, $v_{\mathrm{r}}$ is the relative velocity of the chips to the auger surface, $v_{s}$ is the absolute velocity of the chips, $\omega$ is the angular velocity of the auger, $n$ is the rotary speed of the auger, $r_{1}$ is the radius of the auger, $\alpha$ is the helix angle of the auger, and $\beta$ is the helix angle of the conveying path of the chips.

Solving Eq. (1) gives

$$
\left\{\begin{array}{l}
v_{\mathrm{l}}=\frac{v \sin \alpha \sin \beta}{\sin (\alpha+\beta)}, \\
v_{\mathrm{t}}=\frac{v \sin \alpha \cos \beta}{\sin (\alpha+\beta)},
\end{array}\right.
$$

where $v_{1}$ is the effective conveying component of the $v_{S}$, and $v_{\mathrm{t}}$ is the rotational component of the $v_{\mathrm{s}}$.

The demand flux of cuttings conveyance under a penetrating speed is given by

$$
Q_{\mathrm{p}}=\pi v_{\mathrm{p}}\left(r_{1}^{2}-r_{2}^{2}\right),
$$

where $v_{\mathrm{p}}$ is the penetrating speed, and $r_{2}$ is the coring radius.

The flux of cuttings conveyance capacity of the auger:

$$
Q_{\mathrm{e}}=\left[\pi r_{1}^{2}-\pi\left(r_{1}-h_{\mathrm{s}}\right)^{2}\right] v_{\mathrm{l}},
$$

where $h_{\mathrm{s}}$ is the thickness of the flight.

The drilling device can drill into the simulant smoothly when the volume of the chips produced by penetration (VCP) equals or is lower than the volume of cuttings conveyance capacity (VCCC), otherwise the drill will be blocked by the chips. To ensure a healthy drilling, the critical state of the conveying process can be expressed as

$$
\rho_{\mathrm{i}} Q_{\mathrm{p}}=\rho_{\mathrm{d}} Q_{\mathrm{e}}
$$

where $\rho_{\mathrm{i}}$ is the density of the in situ simulant, and $\rho_{\mathrm{d}}$ is the density of the chips.

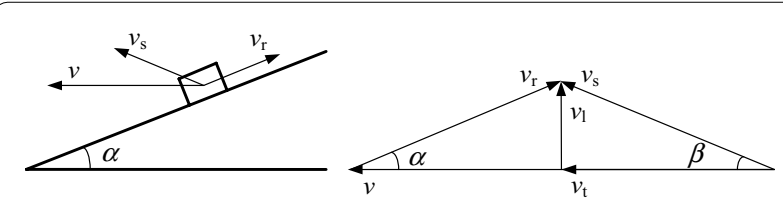

Figure 3 Kinematic analysis of the conveying action 
To obtain $\beta$, the mechanical analysis of the conveying action is carried out as shown in Figure 4. $F_{\mathrm{f} 3}$ has two components: friction between the chips and in situ simulant, and cohesion of the chips. Because the chips in the auger groove are under a high-flow condition, they can be regarded as a Molerus I soil, and the cohesion of the chips can be ignored. In the ideal particle conveying action, the forces are in equilibrium condition:

$$
\left\{\begin{array}{l}
F_{\mathrm{f} 3}=N_{2} \tan \phi, \\
F_{\mathrm{f} 3} \cos (\alpha+\beta)=F_{\mathrm{f} 1}+F_{\mathrm{f} 2}+G \sin \alpha, \\
F_{\mathrm{f} 3} \sin (\alpha+\beta)+G \cos \alpha=N, \\
F_{\mathrm{f} 1}=N_{2} \tan \varphi, \\
F_{\mathrm{f} 2}=N \tan \varphi,
\end{array}\right.
$$

where $F_{\mathrm{f} 3}$ is the frictional force between the chips and the in situ simulant, $F_{\mathrm{f} 1}$ is the friction force between the chips and the auger groove inner surface, $F_{\mathrm{f} 2}$ is the friction force between the chips and the auger blade, $N$ is the supporting force from the auger blade, $N_{2}$ is the supporting force that is vertical to the interface between the chips and the in situ simulant, $G$ is the gravity of the chips, $\phi$ is internal friction angle of the cuttings, and $\varphi$ is the helix angle of the conveying path of the chips.

Solving Eq. (3) gives

$\int_{s} \frac{\delta_{y}}{\delta_{z 1}+\delta_{z 2}} \cos (\alpha+\beta) \tan \phi \mathrm{d} s=\int_{s} \frac{\delta_{y}}{\delta_{z 1}+\delta_{z 2}} \tan \varphi \mathrm{d} s+$

$\int_{s}\left\{g h_{s} \cos \alpha+\frac{\delta_{y}}{\delta_{z 1}+\delta_{z 2}} \sin (\alpha+\beta)\right\} \tan \varphi \mathrm{d} s+\int_{s} g h_{s} \sin \alpha \mathrm{d} s$,

where $g$ is the gravitational acceleration, $\delta_{\mathrm{z} 1}$ is the top normal stress as shown in Figure 5, $\delta_{\mathrm{z} 2}$ is the bottom normal stress, $\delta_{\mathrm{y}}$ is the lateral normal stress, $h$ is the height of the auger groove as shown in Figure 5 , and $\mathrm{d} s$ is the infinitesimal length along the helix of the auger.

To simplify the modeling process, the drill-soil interaction model is developed in the critical state. According to Eqs. (2), (4), and (7), the conveyance analysis flowchart is shown in Figure 6: (1) using the parameters of the drill tool (as listed in Table 1), drilling parameters, and the shear strength of the simulant as inputs, calculate $Q_{\mathrm{p}}$, (2) assign the initial value of $\rho_{\mathrm{d}}$, and then calculate $Q_{\mathrm{e}}$, (3) search the appropriate value of the $\rho_{\mathrm{d}}$ until Eq. (7) is

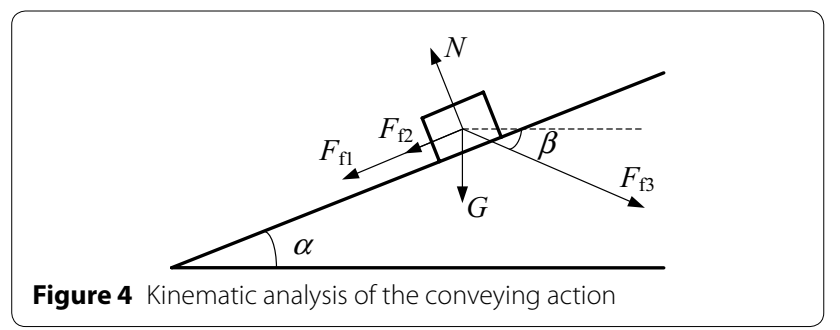

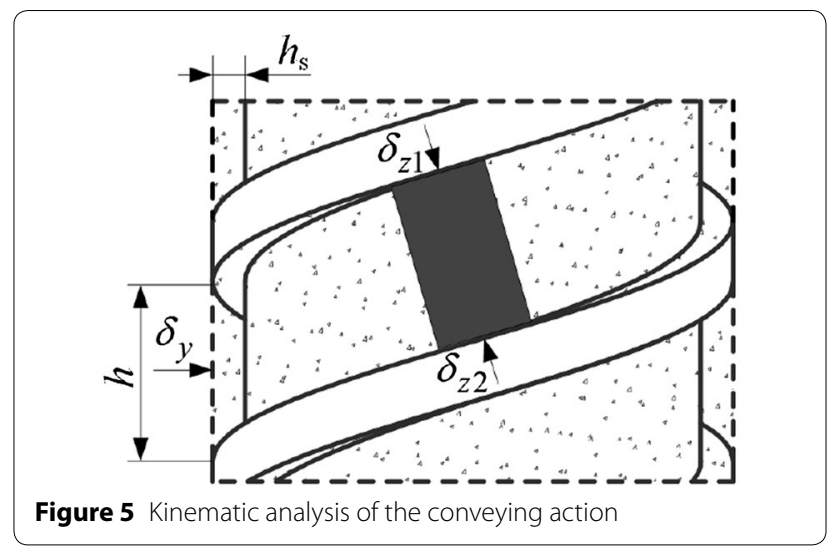

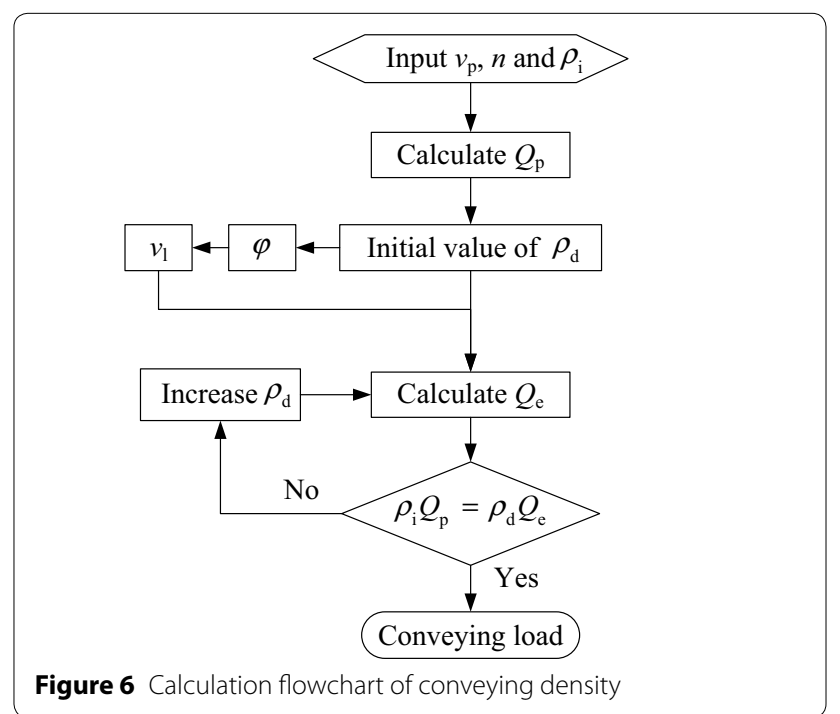

Table 1 Parameters of the drill tool

\begin{tabular}{ll}
\hline Parameter & Value \\
\hline Radius of the auger $r_{1} / \mathrm{mm}$ & 32 \\
Thickness of the flight $h_{s} / \mathrm{mm}$ & 10.53 \\
Height of the flight $h / \mathrm{mm}$ & 2 \\
Helix angle of the auger $a /\left(^{\circ}\right)$ & 14 \\
\hline
\end{tabular}

satisfied, and (4) calculate the conveyance load according to Eq. (7).

The calculated results reveal a trend of higher cuttings conveyance density for higher ratio of penetrating speed to rotary speed (RPS), as shown in Figure 7. The VCP increases with the penetrating speed; and the VCCC is proportional to the rotary speed. As the VCP remains unchanged, the effective conveying speed decreases with the rotary speed. To balance the VCP and keep the healthy drilling, the conveying density and $\beta$ increase 


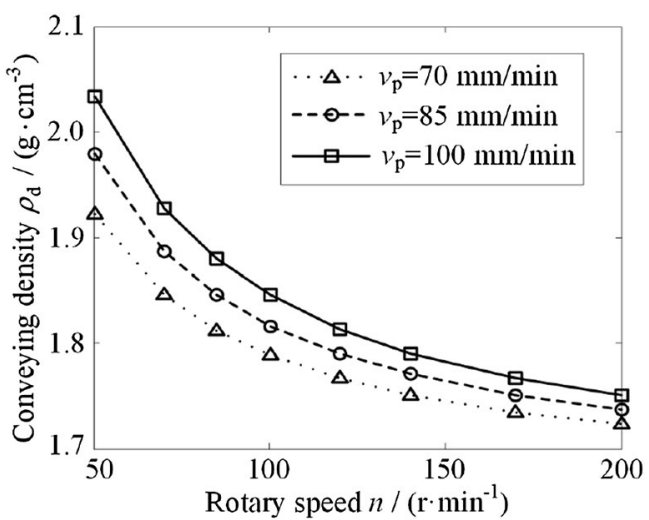

Figure 7 Relation between conveying density and drilling parameters

to keep the VCCC constant. When the RPS remains unchanged, the increase in the VCP is proportional to the increase in the VCCC, resulting in unchanged conveying density. Therefore, the conveying density is determined by the RPS according to the conveying analysis.

Figure 8 shows the relation between the conveying load and the drilling parameters. As the RPS increases, the higher conveying density leads to the increase of the friction between the chips and in situ simulant, resulting in the increase of the conveying load. According to the
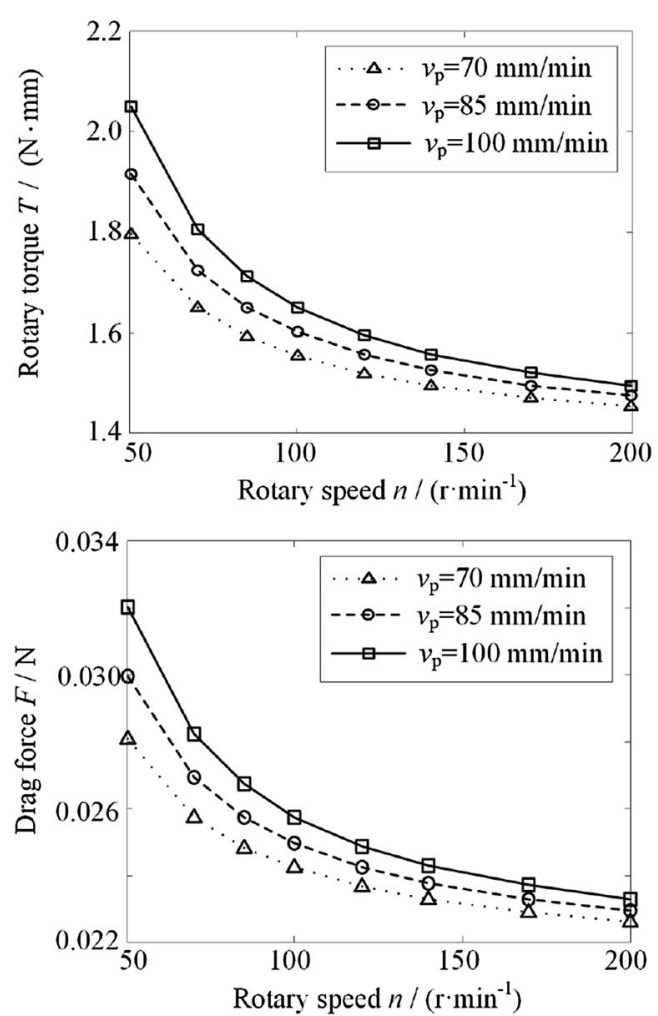

Figure 8 Relation between conveying load and drilling parameters mechanical analysis of the conveying action depicted in Figure 4, the auger withstands tension. Previous experiments indicated that the drill tool withstands pressure rather than tension. Moreover, the rotary torque of the calculated results is normally of $1.4-2.2 \mathrm{~N} \cdot \mathrm{mm}$, which is three orders smaller than the order of the experimental results $(0.1-1 \mathrm{~N} \cdot \mathrm{m})$. Therefore, the conveying load is not the main drilling load during simulant drilling and coring. To predict the drilling load, the next section involves the division of the drill-simulant interaction into four parts, and subsequently, analyzing the drilling load in each part.

\section{Modeling of Drill-Simulant Interaction}

Based on the screw conveying analysis, this section involves the development of the bit-simulant interaction model. Summarizing the analysis of the screw conveying process and bit-simulant interaction, the drill-simulant interaction model can be developed to predict the simulant drilling load, considering the boundary conditions between these two parts. According to the structure of the drill bit, the bit-simulant interaction is divided into three parts (as shown in Figure 9): chip extruding, chip bulldozing, and in situ simulant cutting. The model of bit-simulant interaction is based on the hypothesis as follows:

(1) The simulant is an isotropic, rigid-plastic material;

(2)Because the penetrating speed and the rotary speed are low, the drilling process is regarded as a quasistatic process.

\subsection{Extruding Load Analysis}

The drill tool conveys the chips to the auger in the extruding part, ensuring that the auger conveys the chips with appropriate conveying density under given drilling

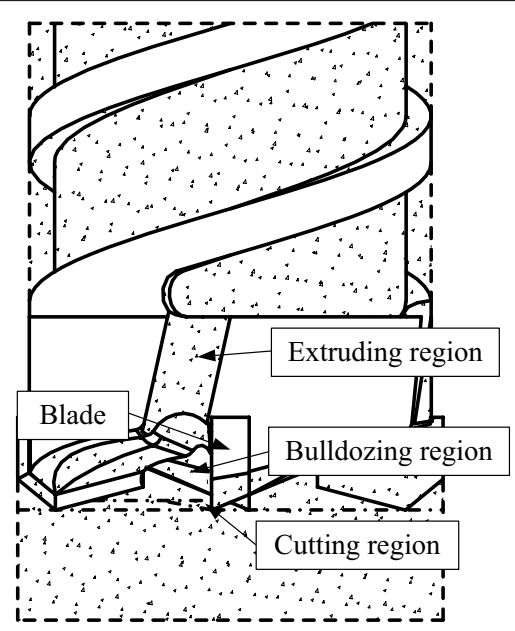

Figure 9 Interaction between drill bit and simulant 
parameters. Because the helix angle in this part is $75^{\circ}$, it is difficult to convey the chips in a screw conveying manner. The model of the extruding part is based on the hypothesis that the chips are extruded to the auger. According to the mechanical analysis of the extruding process (as shown in Figure 10), the force equilibrium equation of the chips in the extruding part is expressed as follows:

$$
\left\{\begin{array}{l}
F_{1}+F_{\mathrm{s}} \cos \left(\alpha_{1}+\beta_{1}\right)=F_{\mathrm{f}}+N_{\mathrm{e}} \tan \varphi+G_{\mathrm{e}} \sin \alpha_{1}, \\
N_{\mathrm{e}}=G_{\mathrm{e}} \cos \alpha_{1}+F_{\mathrm{s}} \sin \left(\alpha_{1}+\beta_{1}\right), \\
F_{\mathrm{s}}=\int \rho g h_{\mathrm{e}} \tan \phi L_{1} \mathrm{~d} s_{\mathrm{e}}, \\
F_{\mathrm{f}}=\int \rho g h_{\mathrm{e}} \tan \varphi L_{2} \mathrm{~d} s_{\mathrm{e}},
\end{array}\right.
$$

where $F_{1}$ is the extruding force, $F_{\mathrm{s}}$ is the friction force between the chips and the in situ simulant, $\alpha_{1}$ is the helix angle of the extruding part, $\beta_{1}$ is the helix angle of the conveying path of the chips in the extruding part, $F_{\mathrm{f}}$ is the friction force between the chips and the drill bit, $N_{\mathrm{e}}$ is the supporting force from the drill bit, $G_{\mathrm{e}}$ is the gravity of the chips in the extruding part, $L_{1}$ is the arc length of the cross section of the drill bit in the extruding part, $L_{2}$ is the arc length of the cross section of the extruding part at the interface between the chips and the in situ simulant, $h_{\mathrm{e}}$ is the height of the extruding part, and $\mathrm{d} s_{\mathrm{e}}$ is the infinitesimal length along the helix of the auger in the extruding part.

To ensure healthy drilling, the volume of the cuttings conveyance capability is equal to the demand volume of the cuttings conveyance under a given penetrating speed. $\beta_{1}$ is determined by the following equation:

$$
\left\{\begin{array}{l}
v_{\mathrm{er}}=\frac{Q_{\mathrm{e}}}{S_{\mathrm{s}} \sin \alpha_{1}}, \\
\beta_{1}=\operatorname{arccot} \frac{v-v_{\mathrm{er}} \cos \alpha_{1}}{v_{\mathrm{er}} \sin \alpha_{1}},
\end{array}\right.
$$

where $v_{\mathrm{er}}$ is the relative velocity of the chips to the drill bit in the extruding part, and $S_{\mathrm{s}}$ is the sectional area of the extruding part.

According to Eqs. (8) and (9), Figure 11 shows the relation between the extruding load and drilling parameters when the penetrating speed is $85 \mathrm{~mm} / \mathrm{min}$. The extruding force of the calculated results is normally of the order

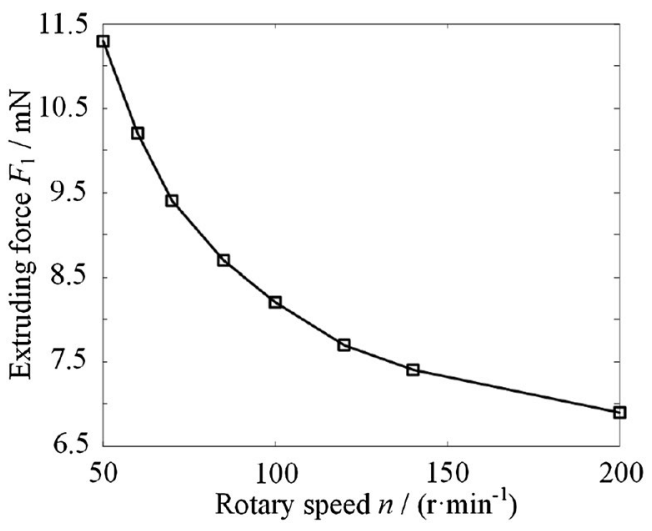

Figure 11 Extruding force with varying drilling parameters

of $7.5-11.5 \mathrm{mN}$, which is three orders smaller than the magnitude of the penetrating force in the drilling experiments (with order of 3-300 N). The following subsection discusses other parts of the bit-simulant interaction.

\subsection{Bulldozing Load Analysis}

The drill bit of the bulldozing part bulldozes the chips, and conveys the chips to the extruding part. The drilling loads of the bulldozing part are generated from the following parts: (1) compression of the loose chips into chips with conveying density by the drill bit, (2) the friction between the drill bit and the chips, and (3) the friction between the chips and the in situ simulant. Because the chips are the Molerus I soil, the analysis of the chips in the bulldozing part is based on the Walker analysis method, as shown in Figure 12 [41].

The compression of the chips in the bulldozing part is similar to the process of confined compression. The force that compresses the loose chips into the chips with conveying density, is determined by confined compression tests, which were conducted using a triaxial test system as shown in Figure 13 (following the SL237-1999 standard procedure [38]). The relation between the compression

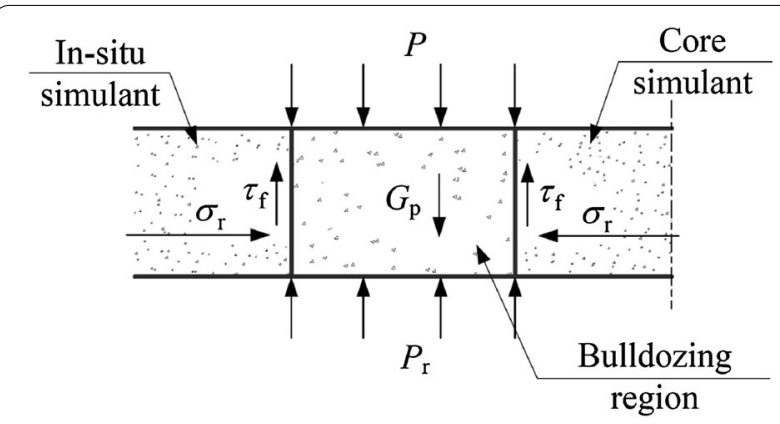

Figure 12 Mechanical analysis of the bulldozing part

Figure 10 Mechanical analysis of the extruding process 


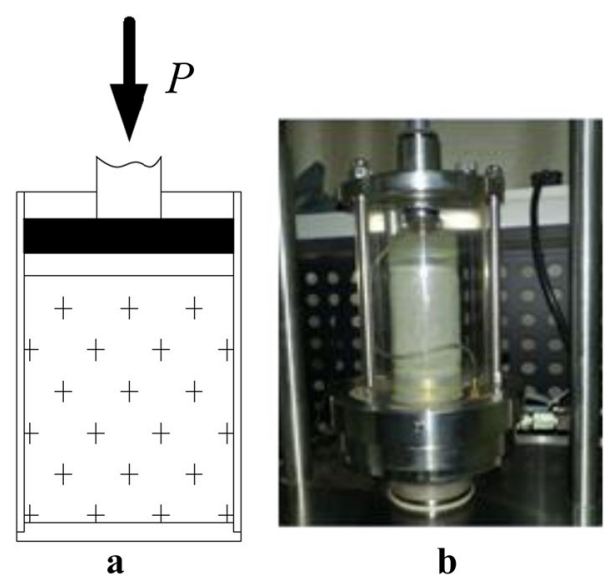

Figure $\mathbf{1 3}$ Triaxial test system

pressure and the density of the simulant density is shown in Figure 14 and can be fitted as follows [23]:

$$
P=1.43 \times 10^{-6} \rho^{28.51},
$$

where $P$ is the compression pressure, and $\rho$ is the density of the simulant.

According to the mechanical analysis of the bulldozing part, the force equilibrium equation of the simulant in the bulldozing part can be expressed as follows:

$$
\left\{\begin{array}{l}
P_{\mathrm{r}} \pi\left(r_{1}^{2}-r_{2}^{2}\right)=P \pi\left(r_{1}^{2}-r_{2}^{2}\right)-\tau_{\mathrm{f}} S_{r 1} \sin \beta_{2}-\tau_{\mathrm{f}} S_{r 2} \sin \beta_{3}+G_{\mathrm{p}}, \\
\sigma_{\mathrm{r}}=k_{\mathrm{k}}\left[P+\frac{G_{\mathrm{p}}}{2 \pi\left(r_{1}^{2}-r_{2}^{2}\right)}\right], \\
\tau_{\mathrm{f}}=\sigma_{\mathrm{r}} \tan \phi \\
\beta_{2}=\operatorname{arccot} \frac{2 \pi r_{1} n}{v_{\mathrm{p}}} \\
\beta_{3}=\operatorname{arccot} \frac{2 \pi r_{2} n}{v_{\mathrm{p}}}
\end{array}\right.
$$

where $P_{\mathrm{r}}$ is the pressure on the bottom of the bulldozing part, $\beta_{2}$ is the penetrating helix angle of the drill bit external cylinder, $\beta_{3}$ is the penetrating helix angle of the drill

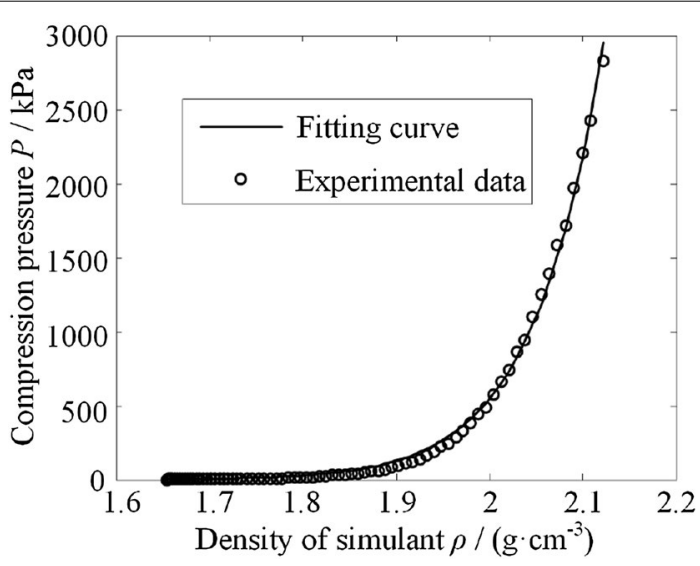

Figure 14 Relation between density and compression pressure bit inner cylinder, $\tau_{\mathrm{f}}$ is the shear stress between the chips and the in situ simulant, $\sigma_{\mathrm{r}}$ is the normal stress, which is vertical to the interface of the chips and the in situ simulant, $S_{\mathrm{r} 1}$ is the external area of the bulldozing part, $S_{\mathrm{r} 2}$ is the internal area of the bulldozing part, and $k_{\mathrm{k}}$ is the Walker constant.

The friction shear stress between the chips and the bit can be expressed as

$$
\tau_{\mathrm{fb}}=\frac{P}{\cos \theta_{1}} \times \tan \varphi .
$$

Summarizing Eq. (11) and Eq. (12), the weight on bit (WOB) of the bulldozing part is expressed as

$$
F_{2}=P \times \pi\left(r_{1}^{2}-r_{2}^{2}\right)+\tau_{\mathrm{fb}} \times \frac{\pi\left(r_{1}^{2}-r_{2}^{2}\right)}{\cos \theta_{1}} \sin \theta_{1} .
$$

The rotary torque of the bulldozing part is expressed as

$$
\begin{array}{r}
T_{\mathrm{b}}=\frac{2}{3} \pi\left(r_{1}^{3}-r_{2}^{3}\right) P_{\mathrm{r}} \tan \phi+k_{\mathrm{k}} \delta_{\mathrm{r}} S_{\mathrm{r} 1} r_{1} \cos \beta_{2}+ \\
k_{\mathrm{k}} \delta_{\mathrm{r}} S_{\mathrm{r} 2} r_{2} \cos \beta_{3}+\frac{2}{3} \pi\left(r_{1}^{3}-r_{2}^{3}\right) \tau_{\mathrm{fb}} .
\end{array}
$$

According to Eqs. (13) and (14), Figure 15 shows the relation between the bulldozing load and the rotary speed when the penetrating speed is $85 \mathrm{~mm} / \mathrm{min}$. As the penetrating speed remains unchanged, the VCP keeps constant. To ensure the healthy drilling, the VCCC should remain unchanged to balance the VCP. When the rotary speed decreases, the conveying density and $\beta$ increase to keep the VCCC constant. The loose chips are compressed into the cuttings with conveyance density by the drill bit in the bulldozing part. The bulldozing load increases with the conveyance density. Therefore, as the penetrating speed keeps constant, a lower rotary speed leads to higher bulldozing load.
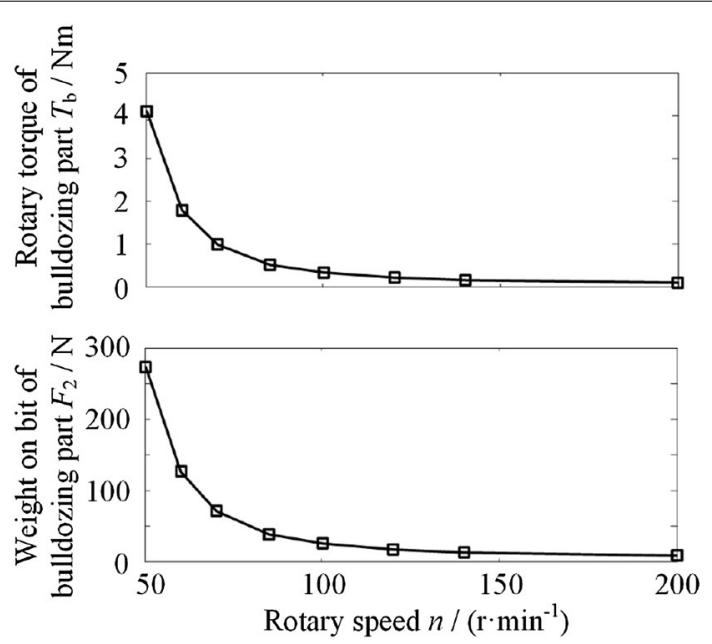

Figure 15 Bulldozing load with varying drilling parameters 


\subsection{Cutting Load Analysis}

The cutting depth of the blade on the drill bit is generally $0-0.5 \mathrm{~mm}$ in the planetary drilling, which is far smaller than the width of the blade [23]. The soil failure can be assumed to take place in a two-dimensional field during the cutting process $[21,23,42]$. Because the simulant is a type of a granular material, shear rupture is the major rupture method during cutting [19-21]. Additionally, the simulant in the cutting part withstands the surcharge load from the bulldozing part. The upper-bound limit method is suitable to calculate the cutting force with the blade rake angle of $90^{\circ}$, and is used to predict simulant cutting force, as shown in Figure 16 [42]. The region ABC is a triangular zone, which is governed by simulant-tool friction. The region ACD is a logarithmic spiral mixed region. The region $\mathrm{ADE}$ is also a triangular zone and is assumed to be a Rankine zone.

In Figure 16, $v_{0}$ is the velocity of the blade, $v_{2}$ is the velocity of $\mathrm{ABC}, v_{3}$ is the velocity of $\mathrm{ACD}, v_{4}$ is the velocity of $\mathrm{ADE}, W_{1}$ is the weight of $\mathrm{ABC}, W_{2}$ is the weight of $\mathrm{ACD}$, and $W_{3}$ is the weight of $\mathrm{ADE}$.

The upper-bound limit model is solved by the principle of virtual work, and can be expressed as follows:

$$
F_{3}=\left(\frac{1}{2} \gamma H^{2} N_{\gamma}+c H N_{c}+q H N_{q}\right) w .
$$

The equivalent factors are as follows:

$$
\begin{aligned}
N_{c}= & \frac{1}{\sin \alpha_{2} \cos \left(\theta+\delta_{1}\right)}\left[\sin \theta+\frac{\cos \left(\theta-\phi_{1}\right)\left(e^{2 \psi \tan \phi_{1}}-1\right)}{\sin \phi_{1}}+\right. \\
& \left.\frac{\cos \left(\theta-\phi_{1}\right) \sin \left(\alpha_{2}-\theta-\psi\right) e^{2 \psi \tan \phi_{1}}}{\cos \left(\alpha_{2}+\phi_{1}-\theta-\psi\right)}\right] \\
& N_{q}=\frac{\cos \left(\theta-\phi_{1}\right) \cos \left(\alpha_{2}-\theta-\psi\right) e^{2 \psi \tan \phi_{1}}}{\sin \alpha_{2} \cos \left(\theta+\delta_{1}\right) \cos \left(\alpha_{2}+\phi_{1}-\theta-\psi\right)}
\end{aligned}
$$

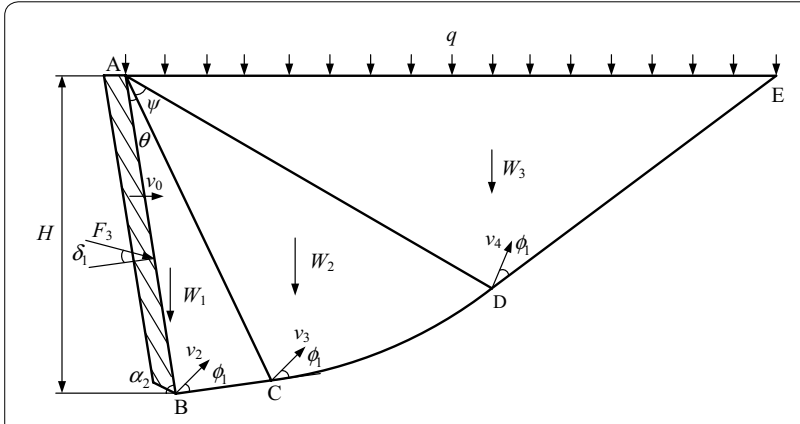

Figure 16 Passive earth pressure analysis of upper-bound limit method

$$
\begin{array}{r}
N_{\gamma}=\frac{\cos \left(\theta-\phi_{1}\right)}{\sin ^{2} \alpha_{2} \cos \left(\theta+\delta_{1}\right) \cos \phi_{1}}\left\{\sin \theta \cos \left(\alpha_{2}-\theta\right)+\right. \\
\frac{\cos \left(\theta-\phi_{1}\right)}{\cos \phi_{1}\left(1+9 \tan ^{2} \phi_{1}\right)} \times\left\{\cos \left(\alpha_{2}-\theta\right) \times\right. \\
{\left[-3 \tan \phi_{1}+e^{3 \psi \tan \phi_{1}}\left(3 \tan \phi_{1} \cos \psi+\sin \psi\right)\right]+} \\
\left.\sin \left(\alpha_{2}-\theta\right)\left[1+e^{3 \psi \tan \phi_{1}}\left(3 \tan \phi_{1} \sin \psi-\cos \psi\right)\right]\right\}+ \\
\left.\frac{\cos \left(\theta-\phi_{1}\right) \cos \left(\alpha_{2}-\theta-\psi\right) \sin \left(\alpha_{2}-\theta-\psi\right) e^{3 \psi \tan \phi_{1}}}{\cos \left(\alpha_{2}+\phi_{1}-\theta-\psi\right)}\right\},
\end{array}
$$

where $F_{3}$ is the cutting force, $H$ is the cutting depth, $\gamma$ is the bulk density of the in situ simulant, $c$ is the apparent cohesion of the in situ simulant, $q$ is the surcharge pressure, $w$ is the width of the bit blade, $\alpha_{2}$ is the rake angle of the blade, $\theta$ is the angle of the region $\mathrm{ABC}, \psi$ is the angle of the region ACD, $\phi_{1}$ is the internal friction angle of the in situ simulant, and $\delta_{1}$ is the angle of simulant-blade friction.

According to the motion of the drill tool, the cutting depth is expressed as

$$
H=\frac{v_{\mathrm{p}}}{n \cdot n_{\mathrm{c}}}
$$

where $n_{\mathrm{c}}$ is the number of blades in the drill bit.

The passive earth pressure is expressed as

$$
E_{\mathrm{p}}=\left(\frac{1}{2} \gamma H^{2} N_{\gamma}+c H N_{c}+q H N_{q}\right) .
$$

Because $\alpha_{2}$ is $90^{\circ}$, the WOB of the cutting part is expressed as

$$
F_{4}=n_{\mathrm{c}} F_{3} \sin \delta_{1} .
$$

The rotary torque of the cutting part is expressed as

$$
T_{\mathrm{c}}=n_{\mathrm{c}} E_{\mathrm{p}}\left(r_{1}^{2}-r_{2}^{2}\right) \cos \delta_{1} / 2 .
$$

According to Eqs. (15), (19), (21), and (22), Figure 17 shows the relation between the cutting force (contains the rotary torque and the WOB of the cutting part) and the rotary speed when the penetrating speed is $85 \mathrm{~mm} /$ min (the regions A and B represent the loads influenced by the surcharge pressure and cutting depth, respectively). As the RPS increases, indicating an increase in the cutting depth and surcharge pressure, the drilling loads increase.

The calculated results indicate that the effect of the surcharge pressure on the cutting force is greater than that of the cutting depth. When the RPS is higher than 0.7, the cutting force caused by the surcharge pressure is the main load. Therefore, the boundary condition has significant effect on the drilling load and must be considered in the modeling process. 


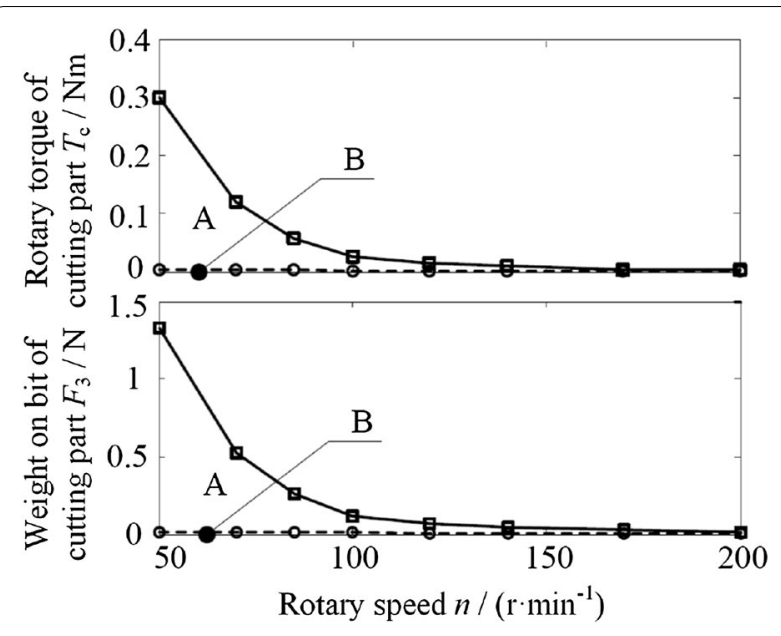

Figure 17 Cutting force with varying drilling parameters

\subsection{Drilling Load Analysis}

Summarizing the aforementioned analysis, the calculation flowchart of the drilling load is depicted in Figure 18, and the parameters of the drill bit are listed in Table 2 . The calculation of the drilling load is based on the screw conveying analysis, and considers the boundary condition among the conveyance part, extruding part, bulldozing part, and simulant cutting part. The bulldozing loads are the major drilling loads, and the cutting loads are secondary, as shown in Figure 19 (the region A represents the bulldozing loads, $B$ represents the cutting loads, and $C$ represents the conveyance and extruding loads). The major part of the WOB is caused by the force that compresses the loose

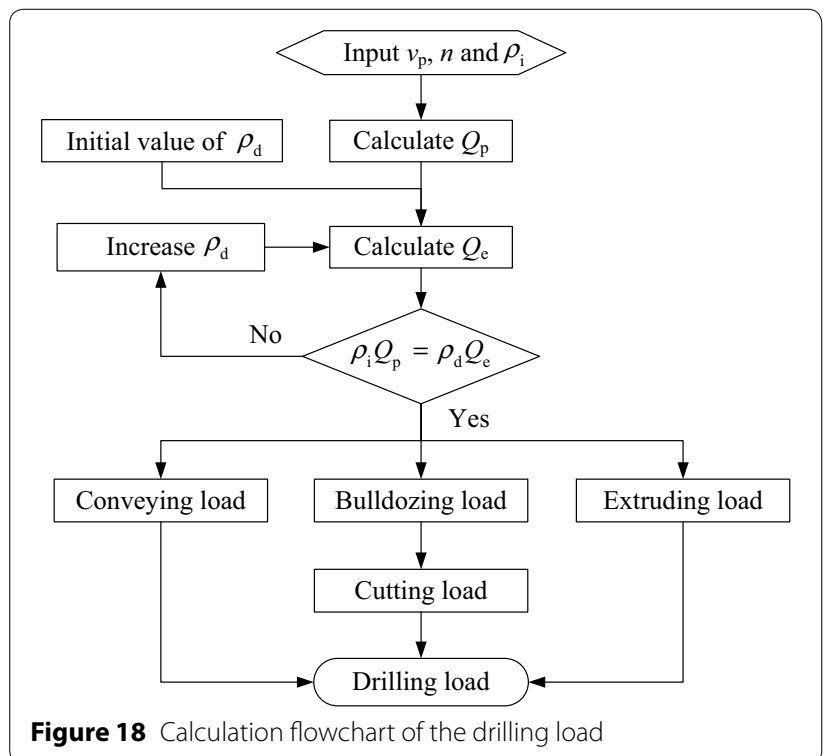

Table 2 Parameters of the drill bit

\begin{tabular}{ll}
\hline Description & Value \\
\hline Height of the extruding part $h_{\mathrm{e}} / \mathrm{mm}$ & 7.4 \\
Width of the bit blade $\mathrm{W} / \mathrm{mm}$ & 10 \\
Helix angle of the extruding part $a_{1} /\left(^{\circ}\right)$ & 75 \\
Rake angle of the blade $\left.a_{2} /{ }^{\circ}\right)$ & 90 \\
Number of the blades $n_{\circ}$ & 4 \\
\hline
\end{tabular}

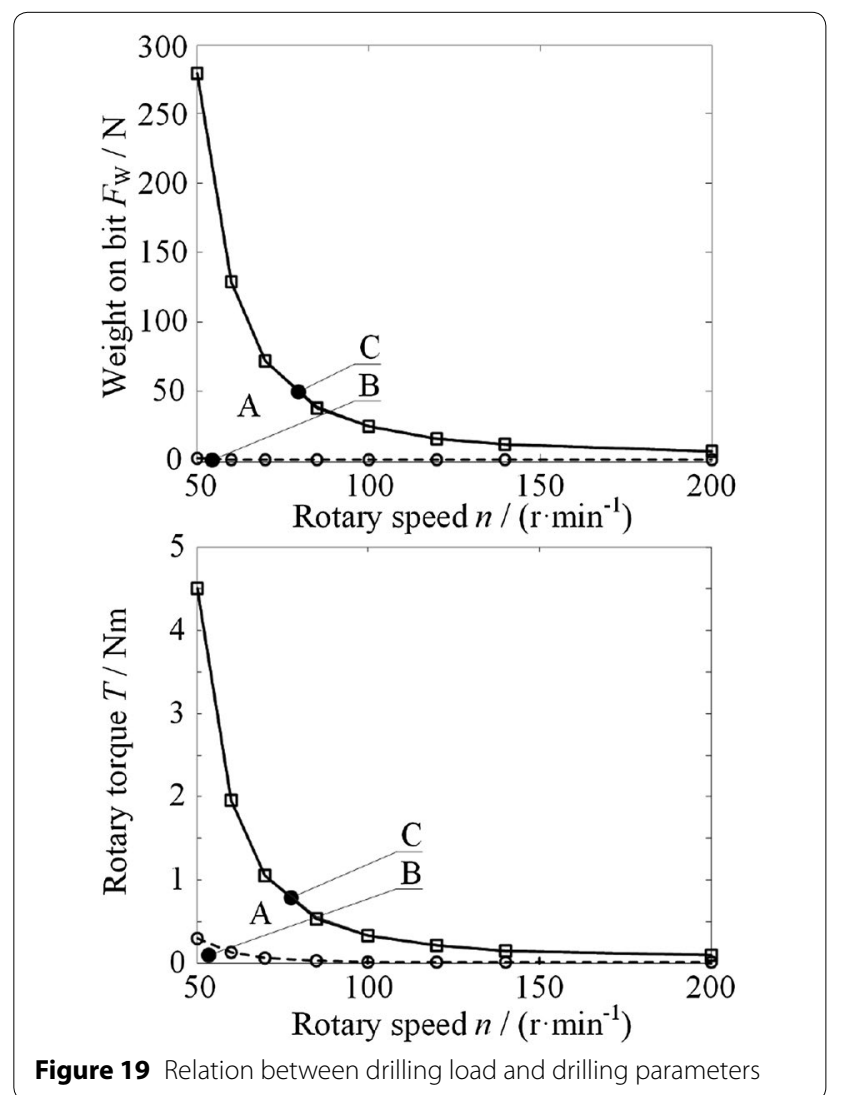

cuttings into the cuttings with conveyance density. The rotary torque is mainly produced by the friction between the cuttings and in situ simulant, and the friction between the drill bit and cuttings in the bulldozing part.

\section{Experimental Verification}

To validate the developed model, experiments were performed using a drilling and coring test-bed equipped with a base of the test-bed, rotary unit, penetrating unit, and lunar regolith simulant bin, as shown in Figure 20. The rotation of the drill is achieved by an AC servo motor with a 7:1 gearhead via a pair of gears with reduction ratio of 60:54. The rotary unit can be vertically actuated along two sliding guides at a desired speed by using a penetrating motor via chains. The control system of the 


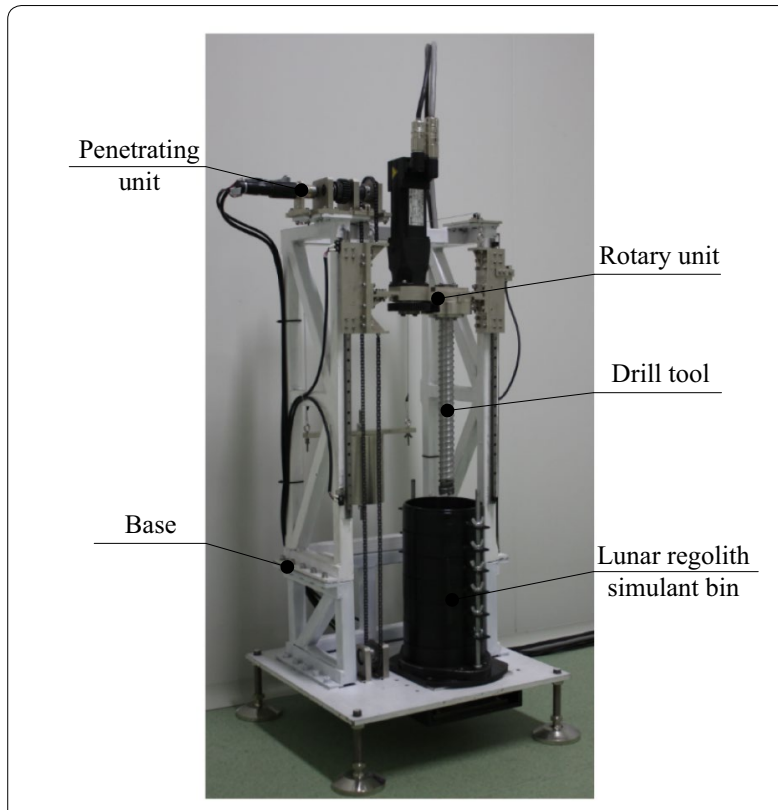

Figure $\mathbf{2 0}$ Drilling and coring test-bed

test-bed collects the real-time data and controls the drilling parameters during the drilling process. The sensors in the test-bed are six-axis force/torque sensors for the rotary torque and $\mathrm{WOB}$, travel switches, and a magnetic scale for penetrating depth. The lunar regolith simulant bin is used to hold the lunar regolith simulant.

Previous experiments (drilling at a length of $0-2.3 \mathrm{~m}$ ) indicated that the drilling loads increase significantly in the shallow layer and approach a steady value at a length of $z>50 \mathrm{~mm}$ (as shown in Figure 21; with the rotary torque and the WOB of $1.2 \mathrm{Nm}$ and 100.0 N). Because the drilling loads are steady in the deep layer, the drilling length for each experiment was $300 \mathrm{~mm}$ from the surface of the lunar soil simulant to make the experiments
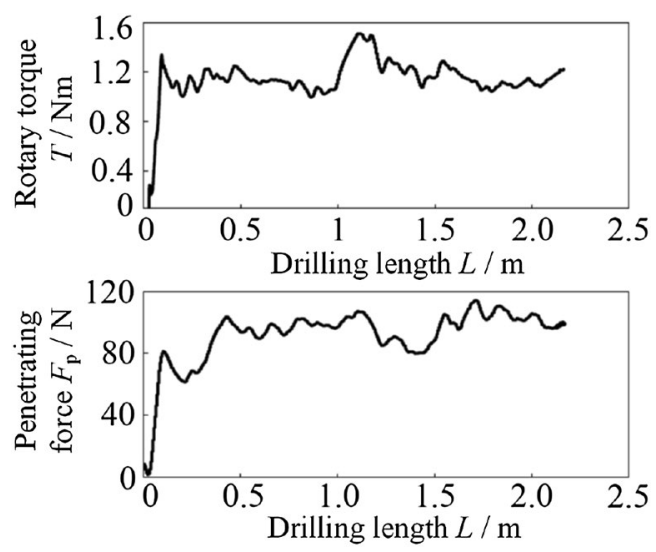

Figure 21 Drilling experiments at a length of 0-2.3 m

more convenient to perform. The simulant sample for each experiment was prepared by five-layer simulant vibratory compaction [33]. The control system collected the sensor data with the nominal sampling rate of $500 \mathrm{~Hz}$. To ensure repeatability of the experiments, each experiment was repeated five times, and the results are presented as their mean value of the steady drilling loads in the deep layer.

The calculated results of the drilling load match well the experimental results, as shown in Table 3. The order of the calculated rotary torque is consistent with the order of the experimental results. As the rotary speed is lower than $200 \mathrm{r} / \mathrm{min}$, the experimental results demonstrate that the drill loads are determined by the RPS; this is consistent with the prediction of the proposed model. When the RPS is higher than 1, the drilling loads increase significantly with the increase of RPS. As the penetrating speed remains unchanged, indicating a constant VCP, a lower rotary speed leads to lower effective screw conveying speed. To balance the VCP, $\beta$ and conveying density

Table 3 Comparison between the experimental and the calculated results

\begin{tabular}{|c|c|c|c|c|c|c|c|}
\hline \multirow[t]{2}{*}{ Penetrating speed $v_{p} /\left(\mathrm{mm} \cdot \mathrm{min}^{-1}\right)$} & \multirow[t]{2}{*}{ Rotary speed $n /\left(r \cdot \min ^{-1}\right)$} & \multicolumn{3}{|c|}{ Weight on bit $F_{w} / N$} & \multicolumn{3}{|c|}{ Rotary torque $T /(\mathrm{N} \cdot \mathrm{m})$} \\
\hline & & Experiments & Average & Model & Experiments & Average & Model \\
\hline 85 & 60 & $61.6-83.6$ & 72.1 & 71.3 & $1.13-1.45$ & 1.31 & 1.05 \\
\hline 85 & 80 & $32.0-41.9$ & 37.7 & 39.0 & $0.70-0.88$ & 0.78 & 0.54 \\
\hline 85 & 100 & $21.2-28.2$ & 25.6 & 24.2 & $0.52-0.59$ & 0.55 & 0.33 \\
\hline 85 & 120 & $15.8-22.2$ & 18.4 & 15.8 & $0.33-0.43$ & 0.37 & 0.21 \\
\hline 85 & 140 & $9.2-17.5$ & 12.7 & 11.6 & $0.22-0.29$ & 0.26 & 0.15 \\
\hline 85 & 200 & $6.6-10.0$ & 7.8 & 6.7 & $0.08-0.16$ & 0.12 & 0.09 \\
\hline 10 & 10 & $32.5-42.0$ & 37 & 39.0 & $0.61-0.8$ & 0.69 & 0.54 \\
\hline 60 & 60 & $36.2-41.9$ & 40 & 39.0 & $0.68-0.78$ & 0.76 & 0.54 \\
\hline 70 & 70 & $34.3-49.5$ & 39 & 39.0 & $0.62-0.80$ & 0.73 & 0.54 \\
\hline 200 & 200 & $32.1-41.5$ & 38 & 39.0 & $0.68-0.72$ & 0.70 & 0.54 \\
\hline
\end{tabular}


of the chips in the auger increase to keep the VCCC constant. According to the analysis of the model, the conveying density of the chips is high when the RPS is higher than 1, resulting in serious bit jamming; this is the cause of high drilling loads. The proposed model can explain the bit jamming during simulant drilling. The experimental and the calculated results indicate that the drilling loads increase smoothly when the RPS is lower than 1. In terms of the drilling load, selection of an appropriate RPS in the range of $0-1$ is suggested when drilling the dense simulant using the drill tool presented in this paper.

\section{Conclusions}

(1) According to the structure of the drill tool, the interaction between the drill tool and lunar regolith simulant is divided into four parts: cuttings screw conveying, chip extruding, chip bulldozing, and in situ simulant cutting. Considering the boundary condition and simulant density difference among these four parts, a drill-simulant interaction model is developed to predict the drilling load during simulant drilling, based on the screw conveying and the passive earth pressure theory.

(2) The mechanical analysis of each part demonstrates that the boundary conditions among these four parts cannot be ignored in the modeling of drill-simulant interaction. According to the analysis of the model, the bulldozing loads include the major drilling loads produced by chip compression, friction between the chips and the in situ simulant, and friction between the drill bit and chips.

(3) A comparison of the drilling experiments and the calculated results demonstrates that the model can effectively predict the drilling load during simulant drilling. With the model, the design of drill tool and development of drilling strategy can be facilitated by a cyclic analysis of drilling load before prototypes are developed for ground drilling tests.

(4) The main mission of the drilling system is to acquire an appropriate sample. While the drilling loads are low when the RPS is lower than 1 , the mass of the coring sample may be low in this range. Combined with the drill-simulant interaction model, in future work, a model for predicting the coring mass should be developed to optimize the drilling parameters.

\section{Authors' contributions}

QQQ and CBC carried out the theoretical analysis and experimental studies and drafted the manuscript. ZQD, JYT, and DWT participated in the sequence alignment and manuscript revision. All authors read and approved the final manuscript.

\section{Authors' Information}

Qi-Quan Quan born in 1983, is currently an associate professor at State Key Laboratory of Robotics and System, Harbin Institute of Technology, China. He received his PhD degree from Ritsumeikan University, Japan, in 2010. His research interests include rotary-percussive ultrasonic drill, Martian UAV, and sensors based on SAW.

Chong-Bin Chen born in 1986, is currently a PhD candidate at State Key Laboratory of Robotics and System, Harbin Institute of Technology, China. He received his master's degree from Nanjing University of Science and Technology, China, in 2012. His area of research includes automated planetary sampling.

Zong-Quan Deng born in 1956, is currently a professor at Harbin Institute of Technology, China. He received his master's degree from Harbin Institute of Technology, China, in 1984. His research interests include planetary rover, deployable space mechanisms, and planetary sampling technology.

Jun-Yue Tang born in 1989, is currently a PhD candidate at State Key Laboratory of Robotics and System, Harbin Institute of Technology, China. He received his master's degree from Harbin Institute of Technology, China, in 2014.

De-Wei Tang born in 1966, is currently a professor at Harbin Institute of Technology, China. He received his PhD degree at Harbin Institute of Technology, China, in 2000. His research interests include in-pipe robots, automated planetary sampling technology.

\section{Acknowledgements}

Supported by National Natural Science Foundation of China (Grant No. 61403106).

\section{Competing interests}

The authors declare that they have no competing interests.

Ethics approval and consent to participate

Not applicable.

\section{Publisher's Note}

Springer Nature remains neutral with regard to jurisdictional claims in published maps and institutional affiliations.

Received: 20 July 2016 Accepted: 14 January 2018

Published online: 27 February 2018

\section{References}

1. Y Bar-Cohen, K Zacny. Extraterrestrial drilling and excavation. Weinheim: Wiley-VCH, 2009.

2. I A Crawford, K H Joy. Lunar exploration: opening a window into the history and evolution of the inner solar system. Philosophical Transactions of the Royal Society A - Mathematical, Physical and Engineering Sciences, 2014, 372: 1-21.

3. F Poletto, P Magnani, R Gelmi, et al. Seismic while drilling (SWD) methodology in support to moon subsurface stratigraphy investigations. Acta Astronautica, 2015, 110: 99-114.

4. T Zhang, Y H An, X L Ding. Device design and implementation method of lunar soil simulant vacuum test. Journal of Beijing University of Aeronautics and Astronautics, 2015, 41(11): 2110-2115. (in Chinese)

5. B J Glass, A Dave, C P Mckay, et al. Robotics and automation for "icebreaker". Journal of Field Robotics, 2014, 31: 192-205.

6. G H Heiken, D TVaniman, B M French. The lunar regolith. Cambridge: Cambridge University Press, 1991.

7. C Pitcher, N Komle, O Leibniz, et al. Investigation of the properties of icy lunar polar regolith simulants. Advances in Space Research, 2016, 57: 1197-1208.

8. X M Shi, Q Q Quan, Z Q Deng, et al. DMT-based estimation of mechanical properties for subsurface lunar soil simulant. Journal of Aerospace Engineering, 2016, 29(4): 04016003-1-04016003-10.

9. A E Finzi, M Lavagna, G Rocchitelli. A drill-soil system modelization for future mars exploration. Planetary and Space Science, 2004, 52: 83-89.

10. TX Liu. Research on lunar soil drilling dynamics based on the discrete element method. Harbin: Harbin Institute of Technology, 2015. (in Chinese)

11. X H Zhu, Y J Jia. 3D mechanical modeling of soil orthogonal cutting under a single reamer cutter based on drucker-prager criterion. Tunnelling and Underground Space Technology, 2014, 41: 255-262. 
12. W Chen, T Qiu. Simulation of earthquake-induced slope deformation using SPH method. International Journal for Numerical and Analytical Methods in Geomechanics, 2014, 38: 297-330.

13. J Wang, D Chan. Frictional contact algorithms in SPH for the simulation of soil-structure interaction. International Journal for Numerical and Analytical Methods in Geomechanics, 2014, 41: 747-770.

14. X M Shi, D G Jie, Q Q Quan, et al. Experimental research on lunar soil simulant drilling load analysis. Journal of Astronaytics, 2014, 35: 648-656. (in Chinese)

15. Y Tian, D W Tang, Z Q Deng, et al. Drilling power consumption and soil conveying volume performances of lunar sampling auger. Chinese Journal of Mechanical Engineering, 2015, 28(3): 451-459. (in Chinese)

16. L L Wang. Parameters optimization and reliability analysis of lunar drilling. Beijing: Beijing Institute of Technology, 2015. (in Chinese)

17. Q Q Quan, J Y Tang, F P Yuan, et al. Drilling load modeling and validation based on the filling rate of auger flute in planetary sampling. Chinese Journal of Aeronautics, 2017, 30: 434-446.

18. Z Q Liu, L L Wang, W R Wu, et al. Effect of structure parameters of drill bit on mechanical performances of lunar automatic drill sampling mechanism. Journal of Astronautics, 2015, 36(12): 1339-1347. (in Chinese)

19. L L Wang, Z Q Liu, W R Wu, et al. Analysis of drill stem structural and kinematic parameters of lunar drilling sampling mechanism. ACTA Aeronautica et Astronautica Sinica, 2016, 37(2): 738-748. (in Chinese)

20. L L Wang, Z Q Liu, W R Wu, et al. Multi-objective optimization of drill stem's motion parameter of lunar automatic drill sampling mechanism. Journal of Astronautics, 2015, 36(6): 723-730. (in Chinese)

21. M Esmaeili, A Ivanovic. Analytical and numerical modelling of non-driven disc on friction material. Computers and Geotechnics, 2015, 68: 208-209.

22. RH King, V Susante, A Gefreh. Analytical models and laboratory measurements of the soil-tool interaction force to push a narrow tool through JSC-1 A lunar simulant and ottawa sand at different cutting depths. Journal of Terramechanics, 2011, 48: 85-95.

23. R J Godwin. A review of the effect of implement geometry on soil failure and implement forces. Soil \& Tillage Research, 2007, 97: 331-340.

24. P D Gupta, C P Gupta, K P Pandey. An analytical model for predicting draft forces on convex-type wide cutting blades. Soil \& Tillage Research, 1989, 14(2): 131-144

25. R J Godwin, M J O’Dogherty. Integrated soil tillage force prediction models. Journal of Terramechanics, 2007, 44(1): 3-14.

26. E Mckyes, O S Ali. The cutting of soil by narrow blades. Journal of Terramechanics, 1977, 14(2): 43-58.
27. A W Roberts, A H Willis. Performance of grain augers. Proceedings of the Institution of Mechanical Engineers, 1962, 176: 165-194.

28. M Mellor. Mechanics of cutting and boring. Part 7. Dynamics and energetics of axial rotation machines. REL Report 81-26, Mississippi: Office of the Chief of Engineers, 1981.

29. K A Zacny, G A Cooper. Methods for cuttings removal from holes drilled on Mars. International Journal of Mars Science and Exploration, 2007, 3: 42-56.

30. A W Roberts. The influence of granular vortex motion on the volumetric performance of enclosed screw conveyors. Powder Technology, 1999, 104: 56-67.

31. J Dai, R Grace. A model for biomass screw feeding. Powder Technology, 2008, 104: 40-55.

32. J C Laul, J J Papike, S B Simon. The Apollo 14 regolith: Chemistry of cores $14210 / 14211$ and 14220 and soils 14141, 14148 and 14149. Journal of Geophysical Research, 1982, 87: A247-A259.

33. C B Chen, Q Q Quan, Z Q Deng, et al. Vibratory compaction method for preparing lunar regolith drilling simulant. Advances in Space Research, 2016, 58: 145-154.

34. J K Mitchell, W N Houston, R F Scott, et al. Mechanical properties of lunar soil: Density, porosity, cohesion, and angle of internal friction. Proceedings of the Third Lunar Science Conference, The MIT Press, 1972, 3: 3235-3253.

35. W D Carrier. Lunar soil grain size distribution. The Moon, 1973, 6: 250-263.

36. J K Mitchell, W N Houston, W D Carrier. Apollo soil mechanics experiment S-200, Final report NASA Contract NAS 9-11266. Berkeley: Space Sciences Laboratory, 1974.

37. TP Gouache, C Brunskill, G P Scott, et al. Regolith simulant preparation methods for hardware testing. Planetary and Space Science, 2010, 58: 1977-1984.

38. The Ministry of Water Resources of the People's Republic of China. SL2371999 Specification of soil test. Beijing: Standards Press of China, 1999.

39. Q Q Quan, C B Chen, Z Q Deng, et al. Recovery rate prediction in lunar regolith simulant drilling. Acta Astronautica, 2017, 133: 121-127.

40. D M Zhao, D W Tang, XY Hou, et al. Soil chip convey of lunar subsurface auger drill. Advances in Space Research, 2016, 57: 2196-2203.

41. R M Nedderman. Statics and Kinematics of Granular Materials. Cambridge: Cambridge University Press, 1992.

42. W F Chen, X L Liu. Limit analysis in soil mechanics. Amsterdam: Elsevier, 1990.

\section{Submit your manuscript to a SpringerOpen ${ }^{\odot}$ journal and benefit from:}

- Convenient online submission

- Rigorous peer review

- Open access: articles freely available online

- High visibility within the field

- Retaining the copyright to your article

Submit your next manuscript at $\boldsymbol{\nabla}$ springeropen.com 